\title{
Relação da qualidade de vida com o estado nutricional e faixa etária de idosos
}

\author{
Aline Bocchi, ${ }^{1}$ Fernanda Scherer Adami ${ }^{2}$
}

\section{Resumo}

O objetivo deste estudo foi relacionar o gênero, a renda, a idade e as medidas antropométricas com os domínios de qualidade de vida dos idosos participantes de um grupo da terceira idade de um município do interior do Rio Grande do Sul. A amostra foi constituída por 103 idosos, que participaram da coleta de dados antropométricos de peso, altura e circunferência da cintura, responderam o questionário validado WHOQOL-BREF e um questionário estruturado sobre dados socioeconômicos, gênero e idade. Foi realizado o cálculo do Indice de Massa Corporal (IMC) e classificado o estado nutricional conforme a Organização Pan-Americana de Saúde (2002) . Quando comparados os domínios de qualidade de vida entre os gêneros, não foi observada associação significativa; em relação ao estado nutricional, 49,5\% dos idosos apresentaram sobrepeso e obesidade. A melhor média foi observada no domínio de meio ambiente, já ao correlacionar as médias dos domínios de qualidade de vida com idade, IMC, circunferência da cintura e peso, observou-se correlação inversa e significativa entre idade e do- mínio físico $(p=0,001)$ e domínio social $(p<0,001)$, bem como entre IMC e domínio psicológico $(p=0,035)$. Peso e circunferência da cintura não foram correlacionados significativamente com os domínios de qualidade de vida. Concluiu-se que, quanto maior a idade, menores foram os escores para os domínios físico e social e, quanto maior o IMC, significativamente menor a média do domínio psicológico.

Palavras-chave: Idoso. Qualidade de vida. Estado nutricional.

\section{Introdução}

O envelhecimento populacional se constitui como um dos principais desafios da atualidade e vem ocorrendo de forma acelerada, sendo que, a cada ano, 650 mil novos idosos são incorporados à sociedade brasileira (SARAIVA et al., 2015). O processo de envelhecimento é inevitável, dinâmico e está associado a

1 Nutricionista egressa do Curso de Nutrição do Centro Universitário Univates - Lajeado (RS). E-mail: alynnebocchi@hotmail.com

2 Graduada em Nutrição pela Universidade do Vale do Rio dos Sinos (2001), especialista em Nutrição Clínica pela mesma instituição (2004), especialista em Educação e Saúde pela Univates (2012), especialista por meritocracia pela Asbran em Saúde Coletiva (2013), mestre em Gerontologia Biomédica pela PUCRS (2008) e doutora em Ambiente e Desenvolvimento pela Univates. Docente e coordenadora do Curso de Nutrição da Univates. E-mail: fernandascherer@univates.br. Endereço para correspondência: Rua Avelino Tallini, 171, Caixa Postal 155, Bairro Universitário, CEP: 95900-000. Lajeado (RS), Brasil.

$\rightarrow$ http://dx.doi.org/10.5335/rbceh.v13i2.5554

Recebido em: 03/12/2015. Aceito em: 06/03/2017. 
modificações físicas, biológicas, psicológicas e sociais, que resultam em dificuldades e incapacidades, aumentando o grau de dependência e comprometendo a funcionalidade e a qualidade de vida do idoso.

Vários aspectos estão relacionados diretamente à qualidade de vida, como a capacidade funcional, o nível socioeconômico, o estado emocional, a interação social, a atividade intelectual, o autocuidado, o suporte familiar, o estado de saúde, os valores culturais, éticos, a religiosidade, o estilo de vida, a satisfação com o emprego e/ou com as atividades da vida diária e com o ambiente em que se vive, vez que cada um desses critérios pode refletir-se de maneira diferente em cada sujeito (BAZANELLA; PICCOLI; QUEVEDO, 2015; DAWALIBI; GOULART; PREARO, 2014; SILVA et al., 2013).

Ao se falar em qualidade de vida na população idosa, torna-se importante conhecer as diferentes situações e realidades nas quais esses indivíduos podem estar inseridos, buscando caracterizar esse fenômeno complexo a inúmeras influências, avaliando a qualidade de vida como uma visão multidimensional (DIAS; CARVALHO; ARAÚJO, 2013). Nos últimos anos, devido ao crescimento da população idosa, o interesse pela monitorização na qualidade de vida aumentou e vem sendo observado em diferentes países devido à sua importância como indicador de saúde pública (NAGAHASHI et al., 2013; CAMPOS et al., 2013).
$\mathrm{O}$ adequado estado nutricional ao longo da vida pode ser considerado um dos fatores que determinam uma velhice bem-sucedida (SOUZA et al., 2013). Possíveis diminuições no peso corporal, estatura e massa muscular, além da redistribuição da gordura corporal, que diminui nos membros e aumenta no tronco, podem ter implicações importantes na saúde do idoso (MENEZES et al., 2013). O estado nutricional inadequado está associado a um variado número de agravos à saúde e, cada vez mais, vem predominando sobre os indivíduos eutróficos, repercutindo indiretamente na qualidade de vida (CINTRA; OLIVEIRA; SILVA, 2012).

O objetivo deste estudo foi relacionar o gênero, a renda, a idade e medidas antropométricas com os domínios de qualidade de vida dos idosos.

\section{Materiais e métodos}

Estudo observacional transversal constituído de uma amostra por conveniência de 103 idosos com idade superior a 60 anos foi realizado no mês de setembro de 2015. O estudo contou com participantes de um grupo da terceira idade do município Doutor Ricardo, localizado no interior do Rio Grande do Sul (RS), com um total de 2076 habitantes, sendo que $21,42 \%$ com 60 anos ou mais de idade (IBGE, 2015; IBGE, 2010). Foram convidados a participar da pesquisa todos os idosos que integravam o grupo, e aqueles que aceitaram o convite assinaram um termo, autorizando a visita da pesquisadora aos encontros, na qual responderam 
o questionário validado WOQOL-BREF e participaram da aferição de peso, altura e circunferência da cintura (CC). Antes da coleta de dados, o Termo de Consentimento Livre Esclarecido foi lido e explicado e, posteriormente, assinado pelos idosos. Este estudo foi aprovado pelo Comitê de Ética em Pesquisa da Univates sob número 459.874/2013.

Para aferir o peso, foi utilizada uma balança digital portátil da marca ZEEX ${ }^{\circledR}$, com capacidade máxima de 150 quilos e com precisão de 100 gramas. Para determinar a estatura, foi usado um estadiômetro portátil PROFISSIONAL $\mathrm{SANNY}^{\circledR}$, com precisão de um milímetro, e a fita métrica da marca CESCORF ${ }^{\circledR}$ com alcance de 2 metros foi utilizada para verificação da CC, que foi classificada conforme a Organização Mundial da Saúde (OMS, 1998). Foi realizado o cálculo do Índice de Massa Corporal (IMC) e, após, classificado o estado nutricional, segundo a Organização Pan-Americana de Saúde (OPAS, 2002). Para determinar a renda dos idosos, categorizou-se a variável em um salário mínimo e mais de um salário mínimo, por ser um ponto de corte da renda mínima salarial brasileira, e a idade foi classificada em duas categorias: 60 a 79 anos e 80 a 99 anos.

Para avaliar a qualidade de vida, foi utilizado o questionário de WHOQOL-BREF que é derivado do WHOQOL-100, sendo este instrumento amplamente utilizado para avaliar a qualidade de vida na velhice devido às suas características tão abrangentes e de simples aplicabilidade. Consiste em 26 questões, que abrangem os domínios físico, psicológico, relações sociais e meio ambiente. Cada domínio é composto por questões cujas pontuações das respostas variam entre 1 e 5 , de acordo com o grau de satisfação (CASTRO; DRIUSSO; OISHI, 2014). Com o instrumento por meio de uma sintaxe que avalia os escores finais de cada domínio, são feitas quatro somas parciais obtidas nos escores de zero a cem, sendo que zero representa a pior qualidade de vida e cem a melhor qualidade (ARIAS; FRANCO; ALZAMORA, 2015).

O domínio físico corresponde a questões relacionadas à dor, ao desconforto, à energia, à fadiga, ao sono, ao repouso, à mobilidade, à atividade de vida cotidiana, à dependência de medicação ou de tratamento e à capacidade de trabalho. O domínio psicológico compreende questões sobre sentimentos positivos, pensamento, aprendizagem, memória e concentração, autoestima, imagem corporal e aparência, sentimentos negativos, espiritualidade, religião e crenças pessoais. O domínio de meio ambiente abrange questões sobre segurança física e proteção, ambiente no lar, ambiente físico (poluição/ruído/trânsito/clima), transporte, recursos financeiros, cuidados de saúde e sociais: disponibilidade e qualidade, oportunidades de adquirir informações e habilidades e de participar em atividades recreativas e de lazer,. O domínio de relações sociais abrange questões sobre relações pessoais, suporte (apoio) social e atividade sexual (GOMES; HAMANN; GUTIERREZ, 2014). Esse questionário é autoaplicável, porém, neste estudo, optou-se pela aplicação em forma de entrevista, dados a 
dificuldade de leitura, problemas visuais e o analfabetismo entre idosos.

Para analisar os dados, foram calculadas as estatísticas descritivas de média e o desvio padrão para as variáveis quantitativas e frequência absoluta e relativa para as variáveis qualitativas. A correlação entre indicadores antropométricos e escores dos domínios do questionário de qualidade de vida foi analisada por meio do coeficiente de correlação de Pearson. As análises estatísticas foram realizadas no Pacote Estatístico Statistical Package for the Social Sience ${ }^{\circledast}$ (SPSS) versão 18.0, sendo considerados significativos valores de $\mathrm{p}<0,05$.

\section{Resultados}

Foram avaliados 103 idosos com idade média igual a 69,80 $\pm 6,61$ anos, sendo a maioria pertencente à faixa etária dos 60-79 anos $(\mathrm{n}=94 ; 91,3 \%)$ e os demais à faixa etária dos 80-99 anos. A amostra foi composta predominantemente por mulheres $(\mathrm{n}=69 ; 67 \%)$. A classificação do estado nutricional, segundo a OPAS, indicou que $49,5 \%$ dos idosos estavam com excesso de peso. Em relação à renda, não foi observada diferença na média do escore de nenhum dos domínios entre aqueles idosos que recebiam um salário mínimo e aqueles que recebiam mais de um salário mínimo. Em relação aos domínios de qualidade de vida entre homens e mulheres não foram observadas diferenças significativas.

$\mathrm{Na}$ Tabela 1, estão descritas as características referentes ao estado nutricional da amostra e a avaliação da qualidade de vida por meio do questionário de WHOQOL-BREF. A maior média foi relacionada ao domínio ambiental, seguida do domínio social, do domínio psicológico e, por fim, do domínio físico, sendo que todos os domínios atingiram médias satisfatórias. Considerando-se a CC como indicador de risco cardiovascular, a maioria dos participantes apresentou risco cardiovascular muito aumentado $(52,4 \%)$ ou aumentado $(28,2 \%)$, e o excesso de peso em relação ao IMC foi constatado em $(49,5 \%)$ dos idosos.

Tabela 1 - Caracterização antropométrica do estado nutricional e da qualidade de vida dos idosos. Doutor Ricardo, 2015 .

\begin{tabular}{|c|c|}
\hline Característica & $\begin{array}{c}\text { Estatística } \\
\text { descritiva }\end{array}$ \\
\hline Peso corporal atual (kg) & $75,55 \pm 12,56$ \\
\hline Estatura (cm) & $162,4 \pm 7,33$ \\
\hline $\mathrm{IMC}\left(\mathrm{kg} / \mathrm{m}^{2}\right)$ & $28,64 \pm 4,34$ \\
\hline Circunferência da Cintura (cm) & $93,53 \pm 10,48$ \\
\hline $\begin{array}{l}\text { Circunferência da Cintura } \\
\text { - classificação } \\
\text { Sem risco CV } \\
\text { Risco CV aumentado } \\
\text { Risco CV muito aumentado }\end{array}$ & $\begin{array}{l}20(19,4 \%) \\
29(28,2 \%) \\
54(52,4 \%)\end{array}$ \\
\hline $\begin{array}{l}\text { Índice de Massa Corporal } \\
\text { — classificação OPAS } \\
\text { Baixo peso } \\
\text { Eutrofia } \\
\text { Sobrepeso } \\
\text { Obesidade }\end{array}$ & $\begin{array}{c}7(6,8 \%) \\
45(43,7 \%) \\
14(13,6 \%) \\
37(35,9 \%)\end{array}$ \\
\hline $\begin{array}{r}\text { Domínio } \\
\end{array}$ & Escore \\
\hline Físico & $61,48 \pm 15,47$ \\
\hline Psicológico & $75,61 \pm 8,93$ \\
\hline Ambiental & $83,55 \pm 7,14$ \\
\hline Social & $81,47 \pm 12,67$ \\
\hline
\end{tabular}

Fonte: primária.

Notas: IMC= Índice de Massa Corporal; CV= Cardiovascular; OMS= Organização Mundial da Saúde; OPAS= Organização Pan-americana de Saúde. Dados apresentados em média e desvio padrão ou frequência absoluta e relativa. 
Na Tabela 2, observou-se correlação inversa e significativa entre idade e domínio físico $(\mathrm{p}=0,001)$ e domínio social $(\mathrm{p}<0,001)$, bem como entre IMC e domí- nio psicológico ( $\mathrm{p}=0,035)$. Peso e CC não foram correlacionados significativamente com os domínios de qualidade de vida.

Tabela 2 - Correlação entre domínios de qualidade de vida e indicadores antropométricos e idade de idosos participantes de um grupo de terceira idade. Doutor Ricardo, 2015.

\begin{tabular}{l|c|c|c|c}
\hline & Domínio Social & $\begin{array}{c}\text { Domínio } \\
\text { Físico }\end{array}$ & $\begin{array}{c}\text { Domínio } \\
\text { Psicológico }\end{array}$ & $\begin{array}{c}\text { Domínio } \\
\text { Ambiental }\end{array}$ \\
\hline Peso & $r=0,087$ & $r=-0,041$ & $r=-0,137$ & $r=0,059$ \\
& $p=0,383$ & $p=0,683$ & $p=0,166$ & $p=0,555$ \\
IMC & $r=0,025$ & $r=-0,033$ & $r=-0,208$ & $r=0,059$ \\
& $p=0,804$ & $p=0,738$ & $p=0,035$ & $p=0,555$ \\
CC & $r=0,041$ & $r=-0,034$ & $r=-0,090$ & $r=0,067$ \\
& $p=0,683$ & $p=0,734$ & $p=0,364$ & $p=0,502$ \\
IDADE & $R=-0,440$ & $R=-0,311$ & $R=0,181$ & $R=-0,065$ \\
& $p<0,001$ & $p=0,001$ & $p=0,067$ & $p=0,513$ \\
\hline
\end{tabular}

Fonte: primária.

Notas: IMC= Índice de Massa Corporal; CC= Circunferência da Cintura. r= Coeficiente de correlação de Pearson. Valores de $\mathrm{p}<0,05$, considerados estatisticamente significativos.

\section{Discussão}

Um estudo realizado por Tavares et al. (2014), com 2143 idosos em Minas Gerais, observou que a maioria da sua amostra teve como renda mensal um salário mínimo, semelhante ao resultado do presente estudo, indicando que a população idosa, em sua maioria, está vivendo com uma renda mínima.

Quando comparados os domínios de qualidade de vida entre os gêneros, não se observou associação significativa, resultado semelhante ao estudo de Modeneze et al. (2013) e Blay e Marchesoni (2011), demonstrando que homens e mulheres apresentam percepções referentes aos domínio similares, diferentemente do estudo de Coelho e Pedroso (2012), no qual o gênero feminino apresentou médias inferiores ao gênero masculino na maioria dos domínios de qualidade de vida avaliados.

Nos estudos de Scherer et al. (2013), com 284 idosos, e Fares et al. (2012), com 477 idosos de Santa Catarina e 316 da Bahia, o estado nutricional da maioria dos idosos foi caracterizado por excesso de peso corporal, resultado semelhante ao encontrado no presente estudo, que também observou que quanto maior a média do IMC significativamente menor foi a média do domínio psicológico. O que sugere que os idosos que apresentam o IMC mais elevado demonstraram menor satisfação em relação às questões sobre os sentimentos positivos e negativos, a aparência e a autoestima (PEREIRA; ALVAREZ; TRAEBERT, 2011). Já no estudo transversal de Wachholz, Rodri- 
gues e Yamane (2011), com 35 idosos do Paraná, não foram encontradas diferenças significativas em nenhum dos domínios, quando comparados ao estado nutricional.

Considerando-se a CC como indicador de risco cardiovascular, a maioria dos participantes da pesquisa apresentou risco cardiovascular aumentado e muito aumentado, semelhante ao estudo de Dawalibi, Goulart e Prearo (2014), realizado com 182 idosos frequentadores de Universidades Abertas de São Paulo. Isso pode estar ocorrendo porque, apesar de o envelhecimento ser um processo natural, o organismo fica submetido a um conjunto de alterações anatômicas e funcionais, sendo essas capazes de modificar o estado nutricional, acarretando em redistribuições de gordura corporal, a qual diminui nos membros e aumenta no tronco, afetando diretamente a vida do idoso (MENEZES et al., 2013; WACHHOLZ; RODRIGUES; YAMANE, 2011; SOUZA et al., 2013). Entretanto, no atual estudo, a média entre peso e CC não foi correlacionada significativamente com os domínios de qualidade de vida. Com esses achados foi possível observar que a média de peso e a $\mathrm{CC}$ não tiveram impacto significativo para aumentar ou diminuir a qualidade de vida da população estudada, diferentemente de Wachholz, Rodrigues e Yamane (2011) que, em seu estudo, observaram que melhores percepções de qualidade de vida correlacionaram-se com estados de sobrepeso, segundo o IMC e Prega Cutânea Tricipital (PCT).
Ao correlacionar as médias dos domínios de qualidade de vida com a idade, observou-se correlação inversa e significativa entre a idade e o domínio físico e social. D. Lima, M. Lima e Ribeiro (2010) referiram, em seu estudo, que o domínio físico demonstrou a menor média entre os domínios, fato que os autores explicam pela relação com a idade mais avançada e, consequentemente, pela impossibilidade de locomoção e de exercer as atividades da vida diária. $\mathrm{Na}$ pesquisa de Gutierrez, Auricchio e Medina (2011), a idade não teve influência nos domínios de qualidade de vida dos idosos participantes da pesquisa, fato que os autores explicam pelo motivo de apenas $3 \%$ dos entrevistados terem idade superior a 80 anos.

Drago e Martins (2012) demonstraram que, quanto maior a idade, mais depressivos se apresentaram os idosos, fator que pode influenciar na percepção da qualidade de vida no domínio social, já que idosos mais velhos e depressivos tendem a participar menos do convívio social.

Estudos feitos por Stival et al. (2014) e Tavares et al. (2014) relataram menor média no domínio de meio ambiente, resultados semelhantes foram obtidos por Rocha et al. (2015), que justificaram este fato pela falta de lazer e pela insatisfação com os serviços de saúde. Esses achados diferem-se dos encontrados neste estudo, no qual a média do domínio de meio ambiente foi a mais alta, e não se observou diferença significativa entre o domínio de meio ambiente e idade, discordando dos resultados de Lacerda et al. (2011), 
que relataram que o domínio de meio ambiente tem associação com a faixa etária e que, quanto menor a idade, pior a qualidade de vida.

Como limitação deste estudo, destaca-se que é necessário considerar que os resultados obtidos são referentes apenas à população de idosos participantes do grupo da terceira idade, portanto os resultados são representativos apenas para os idosos participantes de grupos de terceira idade deste município.

\section{Conclusão}

Com o presente estudo foi possível concluir que as melhores médias em relação à qualidade de vida foram nos domínios de meio ambiente e social. A idade foi inversa e significativamente correlacionada com o domínio físico e social, sendo que, quanto maior a idade, menores os escores para esses domínios. Verificou-se que a maior parte da amostra de idosos apresentou sobrepeso e obesidade e que, quanto maior a média do IMC, menor foi a média do domínio psicológico.

\section{Quality of life of relation with nutritional status and age of elderly}

\section{Abstract}

The objective of this study was to relate gender, income, age and anthropometric measurements with the elderly quality of life domain from a senior group located in a town in Rio Grande do Sul. The sample was built with 103 elderly people, which participated in the collection of the anthropometric data of weight, height and waist circumference, answered the validated questionnaire WHOQOL-BREF and the structured questionnaire about socioeconomic data, gender and age. The Body Mass Index (BMI) was calculated and the nutritional status was classified according to the Pan-American Organization of Health (2002). When comparing the quality of life domain between genders it was not found significant association, when related with the nutritional status, $49,5 \%$ of the elderly revealed overweight and obesity. The best average observed was in the environment domain, but as to correlate the average of the quality of life domain with age, BMI, waist circumference and weight, it was observed the inverse and significant correlation between age and physical domain $(p=0,001)$ and social domain $(p<0,001)$, as between BMI and psychological domain $(p=0,035)$. Weight and waist circumference were not significantly correlated with the quality of life domains. It was concluded that as the superior the age was, the minor were the scores for the physical and social domains, and the superior the BMI was, significantly minor the average of the psychological domain was.

Keywords: Aged. Quality of life. Nutritional status.

\section{Referências}

ARIAS, J. A. C.; FRANCO, L. C. O.; ALZAMORA, A. P. E. Validez discriminante, convergente/divergente, fiabilidad y consistencia interna, del WHOQOL-BREF y el MOSSF-36 en adultos sanos de un municipio colombiano. Revista Facultad Nacional de Salud Pública, Medellín, v. 33, n. 1, p. 50-57, jan./abr. 2015.

BAZANELlA, N. A. L.; PICCOLI, J. C. J.; QUEVEDO, D. M. Qualidade de vida percebida e atividade física: um estudo em 
idosas acima de 80 anos participantes de um programa municipal da saúde da terceira idade na Serra Gaúcha, RS. Estudos Interdisciplinares Sobre o Envelhecimento, Porto Alegre (RS), v. 20, n. 1, p. 249-270, abr. 2015.

BLAY, S. L.; MARCHESONI, M. S. M. Association among physical, psychiatric and socioeconomic conditions and WHOQOL-Bref scores. Cadernos de Saúde Pública. Rio de Janeiro (RJ), v. 27, n. 4, p. 677-686, abr. 2011.

CAMPOS, M. O. et al. Impacto dos fatores de risco para doenças crônicas não transmissíveis na qualidade de vida. Ciência Saúde Coletiva, Rio de Janeiro (RS), v. 18, n. 3, p. 873-882, jan./mar. 2013.

CASTRO, P. C.; DRIUSSO, P.; OISHI, J. Validade convergente entre o SF-36 e o WHOQOL-BREF em idosos. Revista Saúde Pública, São Paulo (SP), v. 48, n. 1, p. 63-67, jan./fev. 2014.

CINTRA, R. M. G.; OLIVEIRA, D.; SILVA, L. M. G. Estado nutricional e ocorrência de hipertensão arterial e de diabetes em idosos residentes e não residentes em instituições geriátricas. Alimentação e Nutrição, Araraquara (SP), v. 23, n. 4 , p. 567-575, out./dez. 2012.

COELHO, E. S. P.; PEDROSO, M. F. B. P. Avaliação da qualidade de vida em idosos residentes no município de Santos/SP. Revista Ceciliana, Santa Cecilia, v. 4, n. 1, p. 04-08, jun. 2012.

DAWALIBI, N. W.; GOULART, R. M. M.; PREARO, L. C. Fatores relacionados à qualidade de vida de idosos em programas para a terceira idade. Revista Ciência \& Saúde Coletiva, Rio de Janeiro, v. 19, n. 8, p. 35053512, jan./ago. 2014.

DIAS, D. S. G.; CARVALHO, C. S.; ARAÚJO, C. V. Comparação da percepção subjetiva de qualidade de vida e bem-estar de idosos que vivem sozinhos, com a família e institucionalizados. Revista Brasileira de Geriatria e Gerontologia, v. 16, n. 1, p. 127-138, 2013.
DRAGO, S. M. M. S.; MARTINS, R. M. L. A depressão no idoso. Millenium - Revista do IPV, n. 43, p. 79-94, jun./dez. 2012.

FARES, D. et al. Fatores associados ao estado nutricional de idosos de duas regiões do Brasil. Revista Associação Médica Brasileira, São Paulo, v. 58, n. 4, p. 434-441, jul./ago. 2012.

GOMES, J. A.; HAMANN, E. M.; GUTIERREZ, M. M. Application of the WHOQOLBREF in a community segment as a subsidy for health promotion actions. Revista Brasileira Epidemiologia, São Paulo, v. 17, n. 2, p. 495-516, abr./jun. 2014.

GUTIERREZ, B. A. O.; AURICCHIO, A. M.; MEDINA, N. V. J. Mensuração da qualidade de vida de idosos em centros de convivência. Journal of the Health Sciences Institute, São Paulo, v. 9, n. 3, p. 186-190, 2011.

INSTITUTO BRASILEIRO DE GEOGRAFIA E ESTATÍSTICA - IBGE. Estimativa da População 2015. Rio de Janeiro, 2015. Disponível em: <http://www.ibge.gov.br/home/ estatistica/populacao/estimativa2015/estimativa_tcu.shtm>. Acesso em: 25 ago. 2016.

INSTITUTO BRASILEIRO DE GEOGRAFIA E ESTATÍSTICA - IBGE. Características da População e dos Domicílios. Censo Demográfico 2010. Rio de Janeiro, 2010. Disponível em: <http://www.sidra.ibge.gov. $\mathrm{br} / \mathrm{cd} / \mathrm{cd} 2010$ universo.asp?o=7\&i=P $>$. Acesso em: 25 ago. 2016.

LACERDA, S. M. et al. Qualidade de vida de idosos atendidos em Programa de Assistência Domiciliária. Revista Brasileira de Geriatria e gerontologia, Rio de Janeiro, v. 14, n. 2, p. 329-342, jan. 2011.

LIMA, D. L.; LIMA, M. A. V. D.; RIBEIRO, C. G. Envelhecimento e qualidade de vida de idosos institucionalizados. Revista Brasileira de Ciências no Envelhecimento Humano, Passo Fundo, v. 7, n. 3, p. 346-356, set./dez. 2010. 
MENEZES, T. N. et al. Perfil antropométrico dos idosos residentes em Campina Grande-PB. Revista Brasileira Geriatria e Gerontologia, Rio de Janeiro, v. 16, n. 1, p. 19-27, jan./mar. 2013.

MODENEZE, D. M. et al. Perfil epidemiológico e socioeconômico de idosos ativos: qualidade de vida associada com renda, escolaridade e morbidades. Estudos Interdisciplinares sobre o Envelhecimento, Porto Alegre, v. 18, n. 2, p. 387-399, dez. 2013.

NAGAHASHI, A. S. et al. Avaliação do estado nutricional, qualidade de vida e prática de atividade física de idosas em programas para terceira idade. Revista Brasileira de Ciências da Saúde, São Paulo, n. 38, p. 38-45, out./ dez. 2013

ORGANIZAÇÃO MUNDIAL DE SAÚDE - OMS. Apresenta texto sobre promoção de saúde. Glossário. Genebra, 1998.

PEREIRA, K. C. R.; ALVAREZ, A. M.; TRAEBERT, J. L. Contribuição das condições sociodemográficas para a percepção da qualidade de vida em idosos. Revista Brasileira de Geriatria e Gerontologia, Rio de Janeiro, v. 14, n. 1, p. 85-95, jan./mar. 2011.

ROCHA, A. C. O. et al. Qualidade de vida de idosos que cuidam de idosos no domicílio. Revista de Enfermagem, Recife, v. 9, n. 2, p. 548-557, fev. 2015.

SARAIVA, A. M. et al. Histórias de cuidado entre idosos institucionalizados: as práticas integrativas como possibilidades terapêuticas. Revista de enfermagem da Universidade Federal de Santa Maria, Santa Maria, v. 5, n. 1, p. 131-140, jan./mar. 2015.

SCHERER, F. et al. Estado nutricional e prevalência de doenças crônicas em idosos de um município do interior do Rio Grande do Sul. Revista Brasileira de Geriatria e Gerontologia, Rio de Janeiro, v. 16, n. 4, p. 769-779, out./dez. 2013.
SILVA, N. A. et al. Força de preensão manual e flexibilidade e suas relações com variáveis antropométricas em idosos. Revista da Associação Médica Brasileira, São Paulo, v. 59, n. 2, p. 128-135, mar./abr. 2013.

SOUZA, R. et al. Avaliação antropométrica em idosos: estimativas de peso e altura e concordância entre classificações de IMC. Revista Brasileira de Geriatria e Gerontologia, Rio de Janeiro, v. 16, n. 1, p. 81-90, jan./ mar. 2013.

STIVAL, M. M. et al. Fatores associados à qualidade de vida de idosos que frequentam uma unidade de saúde do Distrito Federal. Revista Brasileira de Geriatria e Gerontologia, Rio de Janeiro, v. 17, n. 2, p. 395-405, 2014.

TAVARES, D. M. S. et al. Quality of life of elderly. Comparison between urban and rural areas. Investigación y Educación en Enfermería, Medellín, v. 32, n. 3, p. 401-413, set./dez. 2014.

WACHHOLZ, P. A.; RODRIGUES, S. C.; YAMANE, R, Estado nutricional e a qualidade de vida em homens idosos vivendo em instituição de longa permanência em Curitiba, PR. Revista Brasileira de Geriatria e Gerontologia, Rio de Janeiro (RJ), v. 14, n. 4, p. 625-635, out./dez. 2011.

WOLD HEALTH ORGANIZATION. Anales da 36 $6^{\underline{a}}$ Reunión del Comité Asesor de Investigaciones en salud. Encuesta multicentrica: salud, bien estar y envejecimiento (SABE) en América Latina y el Caribe. Washington (DC): Wold Health Organization, 2001. 